\title{
Classical integrable lattice models through quantum group related formalism
}

\author{
Anjan Kundu \\ Fachbereich 17-Mathematik/ Informatik, GH-Universität Kassel \\ Holländische Str. 3634109 Kassel, Germany \\ Permanent address: \\ Saha Institute of Nuclear Physics, AF/1 Bidhan Nagar, \\ Calcutta 700 064,India.
}

\begin{abstract}
We translate effectively our earlier quantum constructions to the classical language and using Yang-Baxterisation of the Faddeev-Reshetikhin-Takhtajan algebra are able to construct Lax operators and associated $r$-matrices of classical integrable models. Thus new as well as known lattice systems of different classes are generated including new types of collective integrable models and canonical models with nonstandard $r$ matrices.
\end{abstract}

\section{Introduction}

The basic aim of present investigation is to show that some effective formalism developed around quantum algebra and quantum integrable systems can also be fruitfully applied to the domain of classical models. In particular we are able to translate most of our results related to the generation of quantum integrable models [1] to the classical language and present a systematic construction of the Lax operators $L(\lambda)$ and related classical $r(\lambda, \mu)$-matrices for new as well as existing lattice models with canonical Poisson-bracket structures. It demonstrates that there exist some fundamental building blocks for both Lax operators and $r$-matrices, out of which these objects can be easily built up following a classical analog of the Yang-Baxterisation of the Faddeev-Reshetikhin-Takhtajan algebra [2].

We also show that applying analogous quantum constructions one can recover cheaply though methodically many classical results, which were possibly discovered originally using deep intuition. Such examples are the famous Ablowitz-Ladik [AL] model [3],discrete time Toda chain [DTTC] [4], asymmetric lattice NLS model [5] etc.. Moreover one can construct now exactly integrable descrete version of derivative NLS and massive Thirrring models, where earlier attempts failed.

Moreover the construction indicates the existence of local as well as global collective integrable models formed by collecting a number of constituent models belonging to the same descendant class, a possibility which has been ignored mostly in lattice model construction. Another new aspect is the formulation of a special class of integrable systems having nonstandared $r$-matrices with both additive and difference dependence on spectral parameters. 
Hopefully the results presented here would draw attention of classical mathematical physicists and thus serve as a bridge narrowing the existing gap between the two camps: classical and quantum.

\section{Building blocks and basic $\mathrm{PB}$ relations}

Let us consider first simple constant solutions of the classical Yang-Baxter equation:

$$
\left[r_{12}, r_{13}\right]+\left[r_{12}, r_{23}\right]+\left[r_{13}, r_{23}\right]=0
$$

given as

$$
r^{+}=\alpha\left(\begin{array}{ccc}
1 & & \\
& 2 & \\
& & 1
\end{array}\right), \quad \text { and } \quad r^{-}=\alpha\left(\begin{array}{ccc}
1 & & \\
& 2 & \\
& & 1
\end{array}\right),
$$

where parameter $\alpha$ may be taken as the deformation parameter in the classical case. Similarly consider $L^{ \pm}$matrices also given in the upper/lower triangular form as

$$
L^{+}=\left(\begin{array}{cc}
\tau_{1}^{+} & \tau_{21} \\
& \tau_{2}^{+}
\end{array}\right), \quad L^{-}=\left(\begin{array}{cc}
\tau_{1}^{-} & \\
\tau_{12} & \tau_{2}^{-}
\end{array}\right),
$$

with elements $\{\tau\}$ being as yet undefined dependent variables. As we see below the matrices $r^{ \pm}$will serve as our building blocks for the construction of spectral parameter dependent classical $r(\xi, \eta)$-matrix, while $L^{ \pm}$will do the same for the related Lax operators $L(\xi)$ of the integrable lattice models. To specify now the nature of $\{\tau\}$ variables these two sets of matrices are linked through the Poisson bracket $(\mathrm{PB})$ relations

$$
\begin{aligned}
& \left\{L^{ \pm} \otimes, L^{ \pm}\right\}=\left[r^{ \pm}, L^{ \pm} \otimes L^{ \pm}\right], \\
& \left\{L^{+} \otimes, L^{-}\right\}=\left[r^{+}, L^{+} \otimes L^{-}\right],
\end{aligned}
$$

which are nothing but the classical analogs of the Faddeev-Reshetikhin-Takhtajan algebra, a well known relation in the subject of quantum group. The relations (2.23 ) in the elementwise form yields the defining $\mathrm{PB}$ relations among the $\tau$ variables as

$$
\begin{array}{r}
\left\{\tau_{12}, \tau_{21}\right\}=2 \alpha\left(\tau_{1}^{-} \tau_{2}^{+}-\tau_{1}^{+} \tau_{2}^{-}\right), \quad\left\{\tau_{i}^{ \pm}, \tau_{j}^{ \pm}\right\}=0, \\
\left\{\tau_{i}^{ \pm}, \tau_{12}\right\}= \pm \epsilon_{i} \alpha \tau_{12} \tau_{i}^{ \pm}, \quad\left\{\tau_{i}^{ \pm}, \tau_{21}\right\}=\mp \epsilon_{i} \alpha \tau_{21} \tau_{i}^{ \pm},
\end{array}
$$

for $i, j=(1,2)$ with $\epsilon_{1}=1, \epsilon_{2}=-1$. Interestingly, when the deforming parameter $\alpha \rightarrow 0$, a consistent limit of (2.4-5) exists with

$$
\left(\tau_{i}^{+}+\tau_{i}^{-}\right) \rightarrow K_{i}^{0}, \quad\left(\tau_{i}^{+}-\tau_{i}^{-}\right) \rightarrow \frac{1}{\alpha} K_{i}^{1}, \quad \tau_{i j} \rightarrow K_{i j}
$$

yielding the PB relations

$$
\begin{array}{r}
\left\{K_{12}, K_{21}\right\}=K_{1}^{0} K_{2}^{1}-K_{1}^{1} K_{2}^{0}, \quad\left\{K_{1}^{0}, K_{2}^{0}\right\}=0, \\
\left\{K_{i}^{0}, K_{12}\right\}=\epsilon_{i} K_{12} K_{i}^{1}, \quad\left\{K_{i}^{0}, K_{21}\right\}=-\epsilon_{i} K_{21} K_{i}^{1},
\end{array}
$$

where $K_{i}^{1}, i=1,2$ becomes central elements with trivial PB with all others. The above Poisson algebras (2.4-5) and (2.7-8), as we show below, will play a decisive role in generating two different large classes of integrable models. 


\section{Lax operators and $r$-matrices through Yang - Baxterisation}

Equipt with all the building materials: $L^{ \pm}, r^{ \pm}$matrices and the relations (2.4-5) or (2.7-8) we can start constructing the Lax operator $L(\xi)$ and the $r(\xi, \eta)$-matrix by stiching together the upper and lower triangular matrices through spectral parameters $\xi, \eta$ as

$$
r(\xi, \eta)=f\left(\xi_{12}\right)\left[\xi_{12}^{-1} r^{+}+\xi_{12} r^{-}\right], \quad L(\xi)=\xi^{-1} L^{+}+\xi L^{-},
$$

where $\xi_{12}=\frac{\xi}{\eta}$ and $f\left(\xi_{12}\right)=\frac{\alpha}{2\left(\xi_{12}^{-1}-\xi_{12}\right)}$ is a function with a pole at $\xi_{12}=1$. To convince ourselves that the $r$ and $L$ matrices thus formed can be considered as the representatives of lattice integrable models, we should check that they satisfy the classical Yang-Baxter equation (CYBE)

$$
\left\{L_{n}(\xi) \otimes, L_{m}(\eta)\right\}=\delta_{m n}\left[r(\xi, \eta), L_{n}(\xi) \otimes L_{n}(\eta)\right] .
$$

This checking however becomes easy due to the algebra (2.2-3) along with the assumption of vanishing $\mathrm{PB}$ at different lattice points (ultralocality condition) and the obvious relation $r^{+}+r^{-}=2 \alpha \mathbf{P}$ through the permutation operator $\mathbf{P}$ ( the classical analog of the Hecke condition ). Therefore one may get from (3.1) (after an irrelevant gauge transformation) a genuine Lax operator and the associated $r$ matrix in explicit form as

$$
L(\xi)=\left(\begin{array}{ccc}
\xi \tau_{1}^{-}+\frac{1}{\xi} \tau_{1}^{+} & \tau_{21} \\
\tau_{12} & \xi \tau_{2}^{-}+\frac{1}{\xi} \tau_{2}^{+}
\end{array}\right),
$$

and

$$
r(\xi, \eta)=\left(\begin{array}{cc}
a(\xi, \eta) & \\
b(\xi, \eta) \\
b(\xi, \eta) \\
& a(\xi, \eta)
\end{array}\right),
$$

where $a(\xi, \eta)=\frac{\eta^{2}+\xi^{2}}{2\left(\eta^{2}-\xi^{2}\right)}$ and $b(\xi, \eta)=\frac{\eta \xi}{\eta^{2}-\xi^{2}}$. Note that expressing spectral parameters in the form $\xi=e^{-\alpha \lambda}, \eta=e^{-\alpha \mu}$ the dependence of the above $r$-matrix (3.4) on trigonometric functions and moreover only on the defference of parameters as $r(\lambda-\mu)$ becomes obvious. Remarkably at deformation parameter $\alpha \rightarrow 0$ due to the existing limit (2.6) of the dependent variables and the expansion of spectral parameter $\xi \approx 1-\alpha \lambda$ the $L(\xi)$ operator (3.3) reduces consistently to

$$
L(\lambda)=\left(\begin{array}{cc}
K_{1}^{0}+\lambda K_{1}^{1} & K_{21} \\
K_{12} & K_{2}^{0}+\lambda K_{2}^{1}
\end{array}\right) .
$$

At the same time due to $f\left(\xi_{12}\right) \rightarrow \frac{\alpha}{2(\lambda-\mu)}$, through the use of the Hecke condition trigonometric $r$-matrix (3.4) reduces to its rational limit

$$
r(\lambda-\mu)=\frac{\mathbf{P}}{\lambda-\mu} .
$$

We show in the next section that the Lax operator (3.3) along with the PB relations (2.4-5) between its elements serves as an excellent ancestor lattice model generating a large class of descendants, all sharing the same trigonometric $r(\xi, \eta)$ matrix (3.4). Similarly (3.5) with (2.7-8) is responsible for another descendant class having the rational form (3.6) for the associated $r$-matrix. 


\section{Classical integrable lattice systems as descen- dant models}

The idea is to insert the Lax operators on a lattice with $n=[1, N]$ sites and find consistent reductions of the general $L$ operator (3.3) with PB relations (2.4-5) or of (3.5) with (2.7-8). For such reductions proper change of dependent variables from $\tau_{n}$ or $K_{n}$ to canonical ones :

$$
\left\{u_{n}, p_{m}\right\}=\delta_{n m} \quad \text { or } \quad\left\{\psi_{n}, \psi_{n}^{\dagger}\right\}=i \delta_{n m}
$$

or to some other physically interesting variables ( like in the AL model), would result different classical integrable lattice systems, since by construction these descendant models would be associated with $r$-matrix (3.4) or (3.6) and satisfy CYBE (3.2).

It is not difficult to show that symmetric reduction of the form

$$
\tau_{1}^{+}=\left(\tau_{2}^{+}\right)^{-1}=-\tau_{2}^{-}=-\left(\tau_{1}^{-}\right)^{-1}, \quad \tau_{12}=\tau_{21}^{\dagger} .
$$

reduces $\mathrm{PB}$ relations (2.4-5) to the classical analog of the $q$-deformed algebra $U_{q}(s u(2))$ [7], while similar symmetric reduction of (2.7-8) as

$$
K_{1}^{1}=K_{2}^{1}=1, \quad K_{1}^{0}=-K_{2}^{0}, \quad K_{12}=K_{21}^{\dagger}
$$

recovers the $s u(2)$ algebra. The reduction (4.1) in turn, expressed through canonical variables $(u, p)$, yields the lattice sine-Gordon model [8], while reduction (4.2) realised in $\left(\psi, \psi^{\dagger}\right)$ gives the standard lattice NLS model [9]. It should be remarked that though such symmetric reductions are important due to their direct relation with popular algebraic structures, the significant feature of our $L$ operators (3.3) and (3.5) is indeed in their explicit asymmetric form. And this in particular allows to yield (without going through any limiting proceedure) the Lax operators of models exhibiting lesser symmetry.

An important example of such asymmetric models related to (3.3) is the Lattice Liouville model with

$$
\tau_{1}^{-}=\tau_{2}^{+}=0, \quad \tau_{1}^{+}=\tau_{2}^{-}
$$

realised through $u, p[1]$. On the other hand asymmetric reduction of (3.5) given by

$$
K_{1}^{1}=i, \quad K_{2}^{0}=K_{2}^{1}=0
$$

with the expression for other functions as

$$
K_{1}^{0}=p, \quad K_{12}=\left(K_{12}\right)^{-1}=e^{u},
$$

yields directly the Toda lattice model.

Remarkably, some recently proposed discrete integrable systems $[5,10]$ can also be derived consistently as other possible asymmetric reductions of (3.5). For example,

$$
K_{2}^{1}=0, K_{2}^{0}=K_{1}^{1}=1, K_{1}^{0}=\phi_{n} \psi_{n}, K_{12}=\psi_{n}, K_{21}=\phi_{n}
$$

with $n=[1, N]$ generates [11] the simple lattice model of [5] , while

$$
K_{2}^{1}=0, K_{2}^{0}=\gamma, K_{1}^{1}=1, K_{1}^{0}=\psi_{i}^{*} \psi_{i}+\omega_{i}, K_{12}=\psi_{i}, K_{21}=\gamma \psi_{i}^{*},
$$


with $i=1,2$ constructs the Toda-like lattice system considered in [10]. Evidently there exist diffrent other reductions of (3.3) and (3.5) capable of generating other integrable systems. We also get as a bonus simultaniously the Lax operators and the $r$ matrices of the constructed models ensuring their integrability.

It is interesting to note that such asymmetries can be made more explicit by considering models with new $r$-matrix solution

$$
\tilde{r}=r_{0}+2 f,
$$

where $r_{0}$ is the original solution (3.4) or (3.6) and

$$
f=\operatorname{diag}\left(\eta^{\prime}-\xi^{\prime}, \eta^{\prime}+\xi^{\prime},-\left(\eta^{\prime}+\xi^{\prime}\right),-\left(\eta^{\prime}-\xi^{\prime}\right)\right)
$$

with colour parameters $\eta^{\prime}, \xi^{\prime}$. The expression (4.6-7) as a new solution of (2.1) is a classical statement of the twisting transformation of [12], which can also be checked otherwise by direct insertion. We consider the particular case

$$
\eta^{\prime}=c \eta+\alpha, \quad \xi^{\prime}=c \xi+\alpha
$$

with $c$ being a constant parameter, which through (4.6-8) yields a new type of $r$ matrix with sum as well as difference dependence on spectral parameters. Observe that at $c=0$, when $f=\operatorname{diag}(0, \alpha,-\alpha, 0)$, with (3.4) as $r_{0}$ one recovers from (4.6) exactly the $r$-matrix associated with the AL [3] as well as the DTTC [4] models. A natural expectation is therefore that these models somehow must be hidden in our construction. To show that this is indeed the case, we note that for this transformed $r$-matrix and with the $L$ operator taken in the form (3.3) the PB relations are changed from (2.4-5) consistently to

$$
\begin{array}{r}
\left\{\tau_{12}, \tau_{21}\right\}=2 \alpha\left(\left(\tau_{1}^{-} \tau_{2}^{+}-\tau_{1}^{+} \tau_{2}^{-}\right)+\tau_{12} \tau_{21}\right) \\
\left\{\tau_{i}^{+}, \tau_{12}\right\}=2 \epsilon_{i} \alpha \tau_{12} \tau_{i}^{+}, \quad\left\{\tau_{i}^{+}, \tau_{21}\right\}=-2 \epsilon_{i} \alpha \tau_{21} \tau_{i}^{+}
\end{array}
$$

with functions $\tau_{i}^{-}$having now trivial $\mathrm{PB}$ with all other elements. The explicit asymmetry of (4.9-10) is obvious, which for

$$
\tau_{1}^{-}=\tau_{2}^{+}=0, \tau_{1}^{+}=\tau_{2}^{-}=1, \tau_{12}=b_{n}, \tau_{12}=b_{n}^{*}
$$

with PB $\left\{b_{m}, b_{n}^{*}\right\}=-2 \alpha\left(1+b_{n} b_{n}^{*}\right) \delta_{m n}$, yields the same AL model. On the other hand reduction of (4.9-10) as

$$
\tau_{1}^{-}=-1, \tau_{2}^{ \pm}=0, \tau_{1}^{+}=e^{\alpha p_{n}}, \tau_{21}=\alpha e^{q_{n}}, \tau_{12}=-\alpha e^{-q_{n}+\alpha p_{n}}
$$

gives the DTTC, meeting our expectation.

\section{Collective models and models with nonadditive $r$-matrices}

An interesting possibility of constructing collective models by joining the constituent integrable elements can be effectively exploited thanks to a symmetry of the CYBE (3.2). In particular, it is easily checked that if $L(\xi, \tau)$ and $\tilde{L}(\xi, \tilde{\tau})$ are Lax operators corresponding to two independent descendant models sharing the same $r$-matrix and with $\{L, \tilde{L}\}=0$, then the collective model $L(\xi, \tau, \tilde{\tau})=L(\xi, \tau) \tilde{L}(\xi, \tilde{\tau})$ will also be 
integrable with the same $r$-matrix. This symmetry allows us to construct integrable models by collecting similar $L$ operators at each lattice point. A recent construction [10] of Toda-like models by joining several bosonic systems is an example of such collective models. A more exciting example is possibly the construction of lattice massive Thirring model by joining integrable discrete derivative NLS models [13]. The discrete derivative NLS in turn may be given by the $L$ operator with reduction of (3.4) as

$$
\begin{aligned}
\tau_{1}^{+} & =\left(\tau_{2}^{+}\right)^{-1}=q^{-N_{n}}, \tau_{1}^{-}=\frac{a}{4 i} q^{N_{n}+1}, \tau_{2}^{-}=-\frac{a}{4 i} q^{-\left(N_{n}+1\right)}, \\
\tau_{12} & =\left(\frac{a}{2}\right)^{\frac{1}{2}} A_{n}=\tau_{21}^{*}, \quad q=e^{\alpha}
\end{aligned}
$$

where $a$ is the lattice constant, $N_{n}=a \psi_{n}^{*} \psi_{n}$, and $A_{n}$ represents a $q$-boson [14] expressed as $A_{n}=\psi_{n} g\left(N_{n}\right), \quad g^{2}=\frac{\left[2 N_{n}\right]_{q}}{N_{n}}$. Using the invariance of (2.4-5) under the exchange of variables

$$
\tau_{1}^{+} \rightleftharpoons \tau_{2}^{-}, \tau_{2}^{+} \rightleftharpoons \tau_{1}^{-}
$$

a similar operator $L^{(2)}$ with another independent component of $\psi$ variable is obtained, yielding the collective model $L_{n}=L_{n}^{(1)} L_{n}^{(2)}$, which is the discrete version of the massive Thirring model.

Extending this idea to more global level we may even insert completely different descendant models at different lattice nodes, preserving only the periodic structure. It seems that such rich possibilities for constructing new classical integrable lattice systems with canonical structures remain mostly unexplored.

Finally we come to another novel construction of a class of lattice models with nonstandard $r$ matrices. For this we have to choose nontrivial $c$ in (4.8), while as $r_{0}$ in (4.6) one may take either the trigonometric (3.4) or the rational form (3.6). The function $f$ naturally brings in new ( sum as well as difference) dependence on spectral parameters. The associated $L$ operator may also be changed accordingly keeping the $\mathrm{PB}$ relations (2.4-5) or (2.7-8) unchanged. Or alternatively, the $L$ operator may again be chosen as (3.3) or (3.5) and get the deformed PB relations from the CYBE. Following the first choice we get [1]

$$
L(\xi)=\left(\tau_{1}^{-} \tau_{2}^{+}\right)^{c \lambda+\alpha} L^{0}(\xi)
$$

$L^{0}$ being the original $L$ operator (3.3) with the trigonometric $r$ matrix. As has been shown above for $c=0$ this system would contain the AL and the DTTC models. Therefore in the general case it should yield new generalised integrable models with $r$ matrix having an interesting nonadditive dependence on spectral parameters and at the same time it would naturally recover all the other classes of integrable models discussed here at different particular cases. Similar construction exist equally for models with rational $r$ matrix.

\section{Conclusion}

Application of quantum group related constructions to classical integrable discrete systems yields intriguing results in model construction. One gets the Lax operators and associated $r$ matrices in a systematic way, for which to our knowledge no other simple prescription exists. This also gives the construction of some collective integrable models as well as models with nonstandard $r$-matrices. Such possibilities are 
worth exploring for generating new type of integrable lattice models.

\section{Acknowledgement}

The author extends his sincere thanks to the organisers of this conference and the Alexander von Humboldt Foundation for support.

\section{References:}

[1] A. Kundu and B. Basumallick 1992 Mod. Phys. Lett. A 7 61;

A. Kundu and B. Basumallick, Coloured FRT algebra and its Yang-Baxterisation

leading to integrable models with nonadditive R-matrix Saha Inst. preprint, April 1993

[2] N.Yu. Reshitikhin, L.A. Takhtajan and L.D. Faddeev 1989 Algebra and Analysis $\mathbf{1} 17$

[3] M.J. Ablowitz and J.F. Ladik 1976 Stud.Appl.Math. 55213

[4] Yu.B. Suris 1990 Phys.Lett. A 145113

[5] I. Mirola, O. Ragnisco and T.G. Zhang A novel hierarchy of integrable lattices, Rome Univ. preprint n. 976, 1993

[6] V.F.R. Jones 19901993 Int. J. Mod. Phys. B4 701

[7] M. Jimbo 1985 Lett. Math. Phys. 20 331;

V.G. Drinfeld 1986 in Proc. ICM (Berkeley) 798

[8] A.G. Izergin and V.E. Korepin 1982 Nucl. Phys. B 205 [FS 5] 401

[9] A.G. Izergin and V.E.Korepin 1981 Sov.Phys.Dokl. 25976 ;

V.O. Tarasov, L.A. Takhtajan and L.D. Faddeev 1983 Teor. Mat. Fiz. 57

163 [10] P.L. Christiansen, M.F. Jorgensen and V.B. Kuznetsov, 1993 Lett. Math. Phys. 29165

[11] O. Ragnisco and A. Kundu A simple lattice variant of NLS equation and its deformation with exact quantum solution., Saha Inst. preprint SINP/TNP/92-15, 1992

[12] N. Reshetikhin 1990 Lett. Math. Phys. 20331

[13] A. Kundu and B. Basumallick 1993 J. Math. Phys. 341052 ;

B. Basumallick and A. Kundu 1992 Phys.Lett. B 287149

[14] A.J. MacFarlane 1989 J. Phys. A22 4581 